\title{
Preparation, Characterization and Antifungal Activity Studies of AgNPs Loaded Silk Fibroin Hydrogels
}

\author{
Rezaul Haque Ansary*, Tomal Roy, Ali Asraf, Sabina Easmin \\ Department of Chemistry, Rajshahi University, Rajshahi, Bangladesh \\ Email address: \\ ansarychem@gmail.com (R. H. Ansary), tomalroy18@gmail.com (T. Roy), asraf.chem@ru.ac.bd (A. Asraf), \\ sabina_easmin@yahoo.com (S. Easmin) \\ ${ }^{*}$ Corresponding author
}

\section{To cite this article:}

Rezaul Haque Ansary, Tomal Roy, Ali Asraf, Sabina Easmin. Preparation, Characterization and Antifungal Activity Studies of AgNPs Loaded Silk Fibroin Hydrogels. American Journal of Nano Research and Applications. Vol. 8, No. 2, 2020, pp. 28-34.

doi: $10.11648 /$ j.nano.20200802.13

Received: May 23, 2020; Accepted: June 10, 2020; Published: June 28, 2020

\begin{abstract}
Silk fibroin hydrogels are promising materials for controlled drug delivery device due to their aqueous process ability, biocompatibility, and biodegradability. The research work is aimed to prepare silk fibroin (SF) hydrogels loaded with Ag nanoparticles and to evaluate its antifungal activities. The Silk fibroin hydrogels were formulated at $37^{\circ} \mathrm{C}$ using $2 \%$ (w/v) silk fibroin aqueous solution either by treating $50 \%(\mathrm{v} / \mathrm{v})$ of ethanol, or $50 \%(\mathrm{v} / \mathrm{v})$ of propanol, or $50 \%$ (v/v) of glycerol, respectively. Above these, the rate of gelation was sufficiently accelerated by addition of glycerol. The silk fibroin hydrogels and prepared silver nanoparticles (AgNPs) were characterized by using Scanning Electron Microscopy (SEM), Thermo Gravimetrical Analysis (TGA). The encapsulation efficiency and release profile of AgNPs were studied by UV-vis spectrometry. The particle size of AgNPs was measured by Malvern Zetasizer Nano and found $93 \pm 5 \mathrm{~nm}$. The encapsulation efficiency and morphology of the hydrogels was affected by the formulation conditions. The in vitro release profile showed an initial burst release of AgNPs followed by controlled release for next 20 hours. The antifungal activity of AgNPs loaded SF hydrogels showed a positive response to Aspergillus Niger pathogen. Therefore, silk fibroin hydrogels might be an effective biopolymeric matrix for antifungal applications.
\end{abstract}

Keywords: Silk Fibroin, Controlled Release, Hydrogels, Nanoparticles, Drug Delivery

\section{Introduction}

Hydrogels based drug delivery systems are engineered materials that use hydrogels as encapsulation of drugs and cells, vehicles for the targeted delivery and controlled release of therapeutic agents [1]. Several applications in pharmaceutical and medical technology are based on dispersions of particles in a fluid or gel phase [2]. In the last three decades, micro and nanoparticles based polymeric systems have been widely explored for controlled and targeted delivery of drugs and antigens in variety of forms (films, gels, capsules, tablets and creams) mainly due to their ability to sustained drug release for long periods of time, enhanced target, low toxicity, easy manipulation/administration [3-6]. The release properties, the load-bearing capacity, the porosity, and the cell-matrix interactions should be considered for the development of a material to be used in controlled drug delivery and also as scaffolds for tissue engineering [7-9] Also, the possibility to adjust the release profile of a polymeric system is important to achieve the therapeutic concentration of the drug [1]. Silk fibroin (SF) is a protein fiber whose level of crystallinity and molecular conformation makes it a good candidate for controlled drug delivery device. Biomaterials for controlled drug delivery systems have to meet several requirements. They need to be biocompatible, biodegradable, non-toxic, cheap and straight forward to process [10-11]. Some natural (starch, dextran, pullulan, pectin, alginate, chitosan, hyaluronic acid, collagen, gelatin, albumin), semisynthetic and synthetic (PEG, MPEG, PLA, PLGA, PLGA-PEG, PCL, PBCA, PIBCA, PBAEs) polymers are commonly used in drug delivery systems $[8,10,12]$. Recently, silk fibroin based new materials such as powders, films, gels, microparticles, and nanoparticles have been explored for 
surgical and biomedical applications [13-15]. Silk fibroin is a biologically derived protein polymer isolated from domestic silkworm (Bombyx mori) cocoons that possesses unique properties that meet all the requirements for biomedical applications. The unique properties of silk fibroin (SF) such as slow biodegradation, superior mechanical properties, favorable process ability in combination with biocompatibility, have fueled wide interest for a variety of applications, ranging from textiles to biomedical use [16].

Bombyx mori cocoon silk consists of hydrophilic 'glue-like' sericin proteins and the hydrophobic structural protein fibroin, which consists of a heavy chain $(\sim 390 \mathrm{kDa})$ and a light chain ( $\sim 25 \mathrm{kDa})$ [17]. Silk fibroin's heavy chain is composed of large hydrophobic blocks, much smaller hydrophilic blocks and two large hydrophilic blocks at the chain ends at the $\mathrm{N}$ and $\mathrm{C}$ termini. Silk fibroin has some advantages over other natural polymers for hydrogels formation, because its chains are capable to physically crosslink, forming intra- and intermolecular hydrophobic interactions and hydrogen bonds, without any chemical modifications. SF aqueous solution is metastable and SF molecules self-assemble naturally over time into stable crystalline $\beta$-sheet fibrils. This transition occurs naturally in SF aqueous solution because stabilized $\beta$-sheet is the most thermodynamically favorable condition which depends on gelation kinetics. Also, the mechanical properties and degradation rate can be fine-tuned by adjusting SF hydrogels processing conditions and crystallinity [18-19]. Regenerated silk fibroin hydrogels display high oxygen permeability, enzyme-immobilization ability, and good compatibility due to high water content and nature of the polymer [20-22]. Because of these properties, silk based hydrogels have been received lots of interest in the field of drug delivery, tissue engineering and cell culture applications. In a study of Seib et al. (2013), chemotherapeutic agent, doxorubicin was successfully encapsulated in self-assembling silk fibroin hydrogels [23]. The in vivo studies showed maximum therapeutic impact of doxorubicin with minimum harmful side effects. Ebrahimi et al. (2018) formulated SF hydrogels based injectable controlled release formulation of risperidone, a second generation antipsychotric drug [24]. The in vitro release of risperidone from hydrogels was continued up to 25 days which confirms the applicability of SF hydrogels as a controlled delivery device. Therefore, the aim of this study was to develop a formulation of Ag nanoparticles loaded silk fibroin hydrogels to achieve controlled release and better efficacy of AgNPs that can be suited to antifungal applications.

\section{Experimental}

\subsection{Materials and Methods}

Matured Bombyx mori silk cocoons were collected from the Bangladesh Sericulture and Training Institute, Rajshahi, Bangladesh. Sodium carbonate, silver nitrate (Thomas Baker Chemicals Limited, Mumbai, India), lithium bromide (Fisher
Scientific Company, USA), petroleum ether, 3-mercaptopropionic acid, sodium borohydride (Merck, Germany) were used in this study.

\subsection{Extraction of Silk Fibroin from Cocoon}

The silk cocoons were cut by a sharp knife and the insects were taken out. Cocoon shells $(5.00 \mathrm{~g})$ white in color of Bombyx mori were boiled with $300 \mathrm{~mL}$ of $0.02 \mathrm{M} \mathrm{Na}_{2} \mathrm{CO}_{3}$ solution for one hour in a $500 \mathrm{~mL}$ beaker to remove the glue like sericin protein. After that another $300 \mathrm{~mL}$ of $0.02 \mathrm{M}$ $\mathrm{Na}_{2} \mathrm{CO}_{3}$ solution was added to the treated fiber. The hot plate was set to $75^{\circ} \mathrm{C}$ and spinning at the speed of $150 \mathrm{rpm}$ for three sessions for three hours each. The fiber was finally washed three times with boiling water and dried in an oven at $70^{\circ} \mathrm{C}$ for 45 minutes. Moreover, the washed fiber was dried by evacuation in vacuum desiccators for 2 days. Finally, petroleum ether had been used to remove any trace substances of the silk fiber. The collected silk fibroin sample was weighed $(3.62 \mathrm{~g})$ and kept in desiccators for further use.

\subsection{Preparation of Silk Fibroin Solution}

To prepare silk fibroin solution, $4 \mathrm{~g}$ dry silk fibers were soaked into $10 \mathrm{~mL}$ of $9.28 \mathrm{M} \mathrm{LiBr}$ solution in a $25 \mathrm{~mL}$ stopper bottle. The hot plate stirrer was set to $75^{\circ} \mathrm{C}$ at the speed of 200 rpm with continuous stirring until total dissolution of fibers occurred. Finally a clear solution was obtained. The fibroin solution was transferred from the bottle into a cellophane dialyzing bag. The bottle was rinsed with $1 \mathrm{~mL}$ of $9.28 \mathrm{M} \mathrm{LiBr}$ solutions and the washing was also added to the dialyzing bag. Firstly, it was taken $200 \mathrm{~mL}$ of distilled water in a $500 \mathrm{~mL}$ beaker. The dialyzing bag was immersed in the beaker in such way that the level of the bag remained below into the level of dialyzing solvent in the beaker. The dialyzing solvent was gently stirred magnetically. The solution in the bag increased due to osmotic pressure during dialysis and the time was recorded from the beginning of dialysis. Aliquots of fibroin solution were withdrawn from the dialyzing bag and transferred into $2.0 \mathrm{~mL}$ sample vials. The dialyzing solvent, water $(200 \mathrm{~mL})$ was changed at different time interval and the time was recorded. The exchanged solvent was transferred to a $250 \mathrm{~mL}$ volumetric flask and the volume was made up to the mark by adding redistilled water. The bulk of dialyzed fibroin solution was kept in a screw wide neck bottle and placed in a refrigerator at $4^{\circ} \mathrm{C}$ for further applications. Then, the refrigerated silk fibroin solutions were centrifuged at 20,000 rpm for 15 minutes and the collected samples were freeze-dried overnight. The dried samples were used further for the preparation of hydrogels.

\subsection{Preparation of Silk Fibroin Hydrogels}

Silk fibroin hydrogels were prepared in different formulations by treating a $2 \%(\mathrm{w} / \mathrm{v})$ silk fibroin aqueous solution with $50 \%(\mathrm{v} / \mathrm{v})$ of ethanol, or $50 \%(\mathrm{v} / \mathrm{v})$ of propanol, or $50 \%(\mathrm{v} / \mathrm{v})$ of glycerol, respectively. The composition of the formulations has been shown in Table 1. The mixed solutions $(20 \mathrm{~mL})$ were put on molds of diameter $4 \mathrm{~cm}$ and 
placed on a thermostatic bath at $37^{\circ} \mathrm{C}$ until gelation occurred. The gel was taken out and washed gently with redistilled water (Figure 1).

\subsection{Synthesis of Silver Nanoparticles (AgNPs)}

At first, $4 \mathrm{~mL}$ of $0.025 \mathrm{M} \mathrm{AgNO}$ solution was taken in a reaction vessel. After that, $200 \mu \mathrm{L}$ of 3-mercaptopropionic acid solution was added drop by drop and a white precipitate was observed. The precipitate was dissolved by adding $0.1 \mathrm{M}$ $\mathrm{NaOH}$ solution with adjusting the $\mathrm{pH}$. Then $0.2 \mathrm{M} \mathrm{NaBH}$ solution was added drop by drop with stirring until the fade yellow solution turned into deep yellow. The prepared AgNPs solution was characterized and encapsulated in silk fibroin hydrogels.

Table 1. Composition and gelation time of silk fibroin hydrogels prepared in different formulation.

\begin{tabular}{|c|c|c|c|c|c|c|}
\hline $\begin{array}{l}\text { Formulation } \\
\text { No. }\end{array}$ & $\begin{array}{l}\text { Volume of silk fibroin } \\
\text { solution }(\mathrm{mL})\end{array}$ & $\begin{array}{l}\text { Volume of ethanol } \\
\text { solution }(\mathrm{mL})\end{array}$ & $\begin{array}{l}\text { Volume of propanol } \\
\text { solution }(\mathrm{mL})\end{array}$ & $\begin{array}{l}\text { Volume of glycerol } \\
\text { solution }(\mathrm{mL})\end{array}$ & $\begin{array}{l}\text { Volume ratio of } \\
\text { fibroin/solvent }\end{array}$ & $\begin{array}{l}\text { Gelation } \\
\text { time (hours) }\end{array}$ \\
\hline F1 & 5 & 15 & - & - & $1: 3$ & 5 \\
\hline F2 & 5 & - & 15 & - & $1: 3$ & 2 \\
\hline F3 & 5 & - & - & 15 & $1: 3$ & 1 \\
\hline $\mathrm{F} 4$ & 10 & 10 & - & - & $1: 1$ & 12 \\
\hline F5 & 10 & - & 10 & - & $1: 1$ & 7 \\
\hline F7 & 15 & 5 & - & - & $3: 1$ & 20 \\
\hline F8 & 15 & - & 5 & - & $3: 1$ & 12 \\
\hline F9 & 15 & - & - & 5 & $3: 1$ & 5 \\
\hline
\end{tabular}

\subsection{Loading of AgNPs in Silk Fibroin Hydrogels}

Among all the above formulations of fibroin hydrogels, the hydrogels prepared with maintaining fibroin-solvent ratio 1:1 showed good integrity. To investigate the loading of AgNPs in silk fibroin hydrogels, the following study was confined to the formulations where fibroin-solvent ratio was 1:1. For the incorporation of AgNPs into silk fibroin hydrogels, $10 \mathrm{~mL}$ of fibroin dialyzed solution $(0.2 \mathrm{~g} / \mathrm{mL})$ was mixed with different volume of AgNPs solution $(0.02 \mathrm{~g} / \mathrm{mL})$ prior to hydrogels formation (Table 2). Then, $10 \mathrm{~mL}$ of $50 \%$ $(\mathrm{v} / \mathrm{v})$ of ethanol, or propanol, or glycerol was added in the above formulations, respectively and the mixture was placed on molds of $4 \mathrm{~cm}$ in diameter and kept on a thermostatic bath at $37^{\circ} \mathrm{C}$ until gelation occurred. The gel was washed gently with redistilled water.

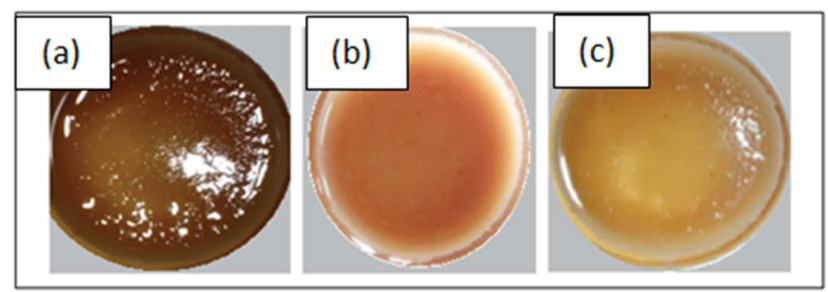

Figure 1. Silk fibroin hydrogels prepared in formulations: (a) F4, (b) F5, (c) F6.

\subsection{Characterizations Techniques}

\subsubsection{Particle Size Analysis}

Average particle size and size distribution of AgNPs was measured by Malvern Zetasizer Nano ZS90 (Malvern, U.K) in the Nanochemistry Lab, Department of Chemistry, Rajshahi University. All the measurements were performed in triplicate. The results were represented as mean \pm standard deviation (S.D).

\subsubsection{Encapsulation Efficiency of AgNPs in Silk Fibroin Hydrogels}

Encapsulation efficiency of AgNPs in silk fibroin hydrogels was conducted by a literature method [25]. Approximately $10 \mathrm{~mL}$ of fibroin dialyzed solution $(0.2 \mathrm{~g} / \mathrm{mL})$ was mixed with $1 \mathrm{~mL}$ of $\operatorname{AgNPs}$ solution $(0.02 \mathrm{~g} / \mathrm{mL})$ prior to hydrogels formation. Then, either $10 \mathrm{~mL}$ of $50 \%(\mathrm{v} / \mathrm{v})$ of ethanol, or propanol, or glycerol was added, and the mixtures were placed on molds of $4 \mathrm{~cm}$ in diameter and kept on a thermostatic bath at $37^{\circ} \mathrm{C}$ until gelation occurred. After hydrogels formation, the supernatant was taken out and analyzed for the determination of residual Ag nanoparticles concentration using a double beam Shimadzu UV-visible spectrophotometer, Model UV-1800 ( $\left.\lambda_{\max }, 360 \mathrm{~nm}\right)$. Standard calibration curve of colloidal $\mathrm{Ag}$ nanoparticles solution was used for AgNPs quantification in the hydrogels. Encapsulation efficiency was determined by the following equation [4]. All the measurements were performed in triplicate. The results were represented as mean \pm standard deviation (S.D).

Encapsulation Efficiency $=\frac{\text { Amount of Ag nanoparticles in hydrogels }}{\text { Ag nanoparticles initially added }} \times 100$

\subsubsection{In Vitro Release Studies of AgNPs from Silk Fibroin Hydrogels}

Approximately $10 \mathrm{mg}$ Silk fibroin hydrogels loaded with AgNPs was immersed in $50 \mathrm{~mL}$ phosphate buffer solution (PBS) at $\mathrm{pH} 7.4$ followed by incubation at $37^{\circ} \mathrm{C}$ with constant shaking at $100 \mathrm{rpm}$. After a periodic intervals, $2 \mathrm{~mL}$ of PBS were collected, replaced by fresh PBS ( $\mathrm{pH} 7.4)$, and analyzed by a double beam Shimadzu UV-visible spectrophotometer, Model UV-1800 $\left(\lambda_{\max }, 360 \mathrm{~nm}\right)$. Standard calibration curve of colloidal Ag nanoparticles solution was used for AgNPs quantification in the hydrogels. All the measurements were performed in triplicate. The results were represented as mean \pm standard deviation (S.D). 
Table 2. Composition of silk fibroin hydrogels loaded with AgNPs prepared in different formulations.

\begin{tabular}{|c|c|c|c|c|c|}
\hline Formulation No. & $\begin{array}{l}\text { Volume of silk fibroin } \\
\text { solution }(\mathrm{mL})\end{array}$ & $\begin{array}{l}\text { Volume of Ag Nps } \\
\text { solution (mL) }\end{array}$ & $\begin{array}{l}\text { Volume of ethanol } \\
\text { solution (mL) }\end{array}$ & $\begin{array}{l}\text { Volume of propanol } \\
\text { solution }(\mathrm{mL})\end{array}$ & $\begin{array}{l}\text { Volume of glycerol } \\
\text { solution (mL) }\end{array}$ \\
\hline F10 & 10 & 1 & 10 & - & - \\
\hline F11 & 10 & 1 & - & 10 & - \\
\hline F12 & 10 & 1 & - & - & 10 \\
\hline F13 & 10 & 2 & 10 & - & - \\
\hline F14 & 10 & 2 & - & 10 & - \\
\hline F15 & 10 & 2 & - & - & 10 \\
\hline F16 & 10 & 3 & 10 & - & - \\
\hline F17 & 10 & 3 & - & 10 & - \\
\hline F18 & 10 & 3 & - & - & 10 \\
\hline
\end{tabular}

\subsubsection{Scanning Electron Microscopy (SEM)}

$20 \mu \mathrm{L}$ of silk fibroin hydrogels was added directly on the top of a conductive tape mounted on a SEM sample stub. The samples were dried overnight in air and then sputtered with platinum. The morphologies of silk hydrogels were imaged at a voltage of $15 \mathrm{KV}$ at room temperature using a Zeiss Supra 55 VP SEM (Carl Zeiss SMT) in the Department of Glass and Ceramics, Rajshahi University of Engineering and Technology.

\subsubsection{Thermo-gravimetric Analysis}

The thermal study of AgNPs loaded silk fibroin hydrogels was observed using a DTG-6H thermogravimetric analyzer (Shimadzu, Japan). The amount of samples for each measurement was about $10 \mathrm{mg}$ and all of the measurements were carried out under a nitrogen atmosphere in the temperature range of $30^{\circ} \mathrm{C}$ to $600^{\circ} \mathrm{C}$ at a heating rate of $10^{\circ} \mathrm{C}$ $\mathrm{min}^{-1}$ in the Central Science Lab, Rajshahi University.

\subsubsection{Antifungal Studies}

The antifungal activity test was carried out according to a reported method [25]. For this purpose, antifungal microbes Aspergillus Niger and Trichoderma were used in this study. The fungus was cultivated on malt extract agar medium and incubated at $30^{\circ} \mathrm{C}$. After 24 hours of incubation, fungal growth was observed and the dimensions of zone around the samples were measured to determine the performance of the samples. Blank hydrogels was used as a control for this study. All the measurements were performed in triplicate.

\section{Results and Discussion}

\subsection{Morphological Studies of Silk Fibroin Hydrogels}

In this study, silk fibroin hydrogels were prepared by adding different contents of dehydrating solvents such as ethanol, or propanol, or glycerol in different contents of silk fibroin dialyzed solutions. It has been reported that the addition of dehydrating solvent in silk fibroin solution alters SF intra and intermolecular interactions resulting in a conformational change from the random coil to $\beta$ sheet [26]. Gelation of silk fibroins occurs due to the conformational change of silk protein. In our study, we first investigated the effect of ethanol, propanol, and glycerol on the gelation time. The time required for the formation of hydrogels was monitored by visual inspection. The hydrogels prepared in formulations F1, F2, and F3 maintaining the fibroin-solvent ratio 1:3 were the most fragile and were difficult to transfer them from the mold. With increasing the content of fibroin solution in formulations F4, F6, and F6 most intact, smooth, and dense hydrogels were formed (Figure 1). The hydrogels do not lose their integrity when kept vertically. The gelation time of the above formulations was $12 \mathrm{~h}, 7 \mathrm{~h}$, and $3 \mathrm{~h}$, respectively. Hydrogels prepared in formulations (F7-F9) with maintaining fibroin-solvent ratio (3:1) had longer time of gelation. The gels were highly rigid, irregular in shape and subsequently collapsed easily. Therefore, the morphology and gelation time of silk fibroin hydrogels is largely affected by fibroin-dehydrating solvent ratio and nature of the dehydrating solvents. Among all the formulations of this study, hydrogels prepared with glycerol used as a cross linker showed considerably reduced time of gelation. Glycerol is a common plasticizer, the presence of glycerol in silk fibroin dialyzed solution could increase the hydrophobicity of the protein which might induce faster gelation. In a study of Hanawa et al. (1995) similar results of reduced gelation time was observed with the addition of glycerol to silk fibroin aqueous solution [26]. The surface morphology of silk fibroin hydrogels was observed by SEM (Figure 2). The image showed smooth, cross-linked structure which might be a result of glycerol accelerated faster gelation process.

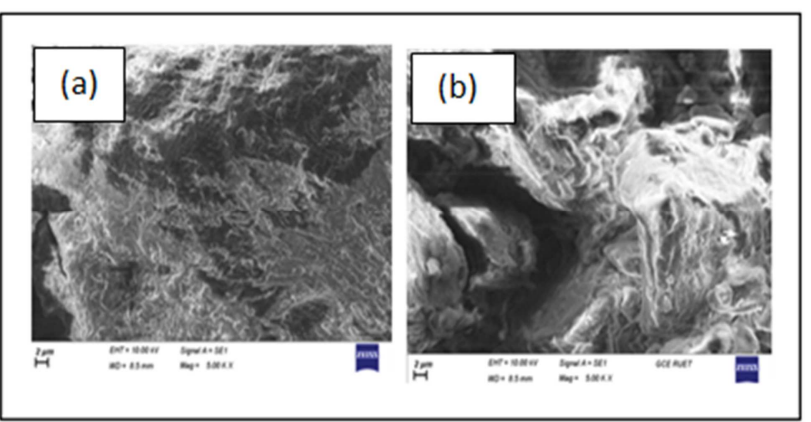

Figure 2. SEM images of silk fibroin hydrogels prepared in formulations (a) F6, (b) F15.

\subsection{Characterization of AgNPs in Colloidal Solution and Silk Fibroin Hydrogels}

The structural characterization of Ag nanoparticles (AgNPs) was performed by UV-visible Spectroscopy [25, 27]. It is quite 
sensitive to quantify colloidal AgNPs using this technique. This is because; colloidal Ag nanoparticles exhibit an intense absorption peak due to the surface plasmon resonances (SPR). The absorption band in the $350-400 \mathrm{~nm}$ regions confirms the formation of $\mathrm{Ag}$ nanoparticles (Figure 3). The average particle size of Ag nanoparticles prepared in our study was measured by dynamic light scattering (DLS) technique and found $93 \pm 5$ $\mathrm{nm}$ with narrow size distribution (Figure 4). In a study of Veerasamy et al., 2010, UV-visible spectra of silver nanoparticles sized $35 \mathrm{~nm}$ exhibited an absorption peak at 438 $\mathrm{nm}$ [28]. It has been reported that with increasing particle size, the optical absorption spectra of metal nanoparticles that are dominated by SPR shift towards longer wave lengths [27]. In order to investigate the applicability of silk fibroin hydrogels as drug delivery system $\mathrm{Ag}$ nanoparticles were loaded in silk fibroin hydrogels that has been described before. The encapsulation efficiency of all the formulations was evaluated thoroughly. The encapsulation efficiency of formulation F13, F14, and F15 demonstrated $73.5 \pm 1.25 \%, 77.3 \pm 2.12 \%$, and $81.4 \pm 1.72 \%$, respectively. The loading of AgNPs increases gradually from ethanol to glycerol. This might be due to the gradual increase in viscosity of alcohols from ethanol to glycerol. Highly viscous glycerol might form strong hydrophobic interactions and hydrogen bonds having three hydroxyl functional groups with the fibroin protein matrix. Therefore, AgNPs might be adhered to the dense surface of the protein matrix through diffusion which resulted in high encapsulation efficiency in formulation F15.

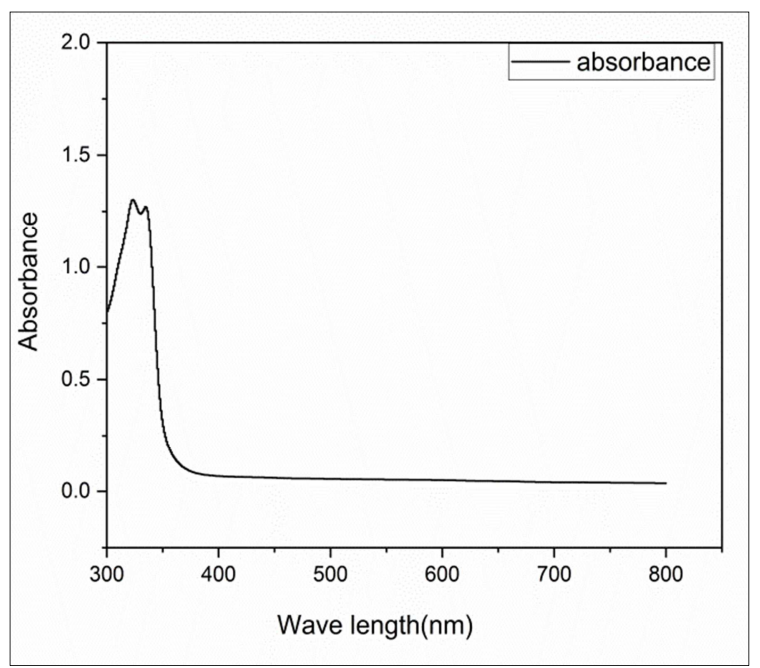

Figure 3. UV-vis spectrum of Ag nanoparticles.

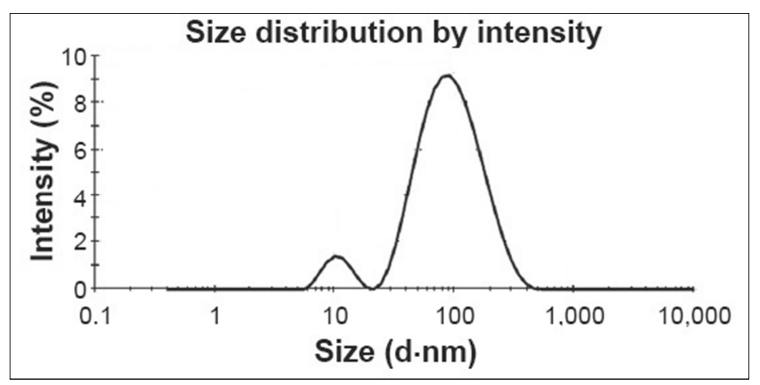

Figure 4. Particle size distribution of Ag nanoparticles.

\subsection{TGA Studies ofAgNPs Loaded Silk Fibroin Hydrogels}

TGA result of AgNPs loaded silk fibroin hydrogels is shown in Figure 5. The result shows that thermal degradation profiles can be divided into two main segments. In segment I, the weight loss was recorded up to $100^{\circ} \mathrm{C}$ which might be due to evaporation of water. The weight loss was continued gradually and a second weight loss was observed between 280 and $380^{\circ} \mathrm{C}$ in segment II. The weight loss occurred due to the breakdown of side chains of amino acid residues and cleavage of peptide bonds [29]. There was a steady weight loss until $600^{\circ} \mathrm{C}$. The total weight loss up to $600^{\circ} \mathrm{C}$ was about $28.51 \%$ as shown in the Figure 5. The TGA curve of AgNPs loaded silk fibroin hydrogels does not exhibit any characteristics differences relative to those of empty hydrogels; this might a be result of relatively low ratio of AgNPs to silk fibroin hydrogels (curve not shown).

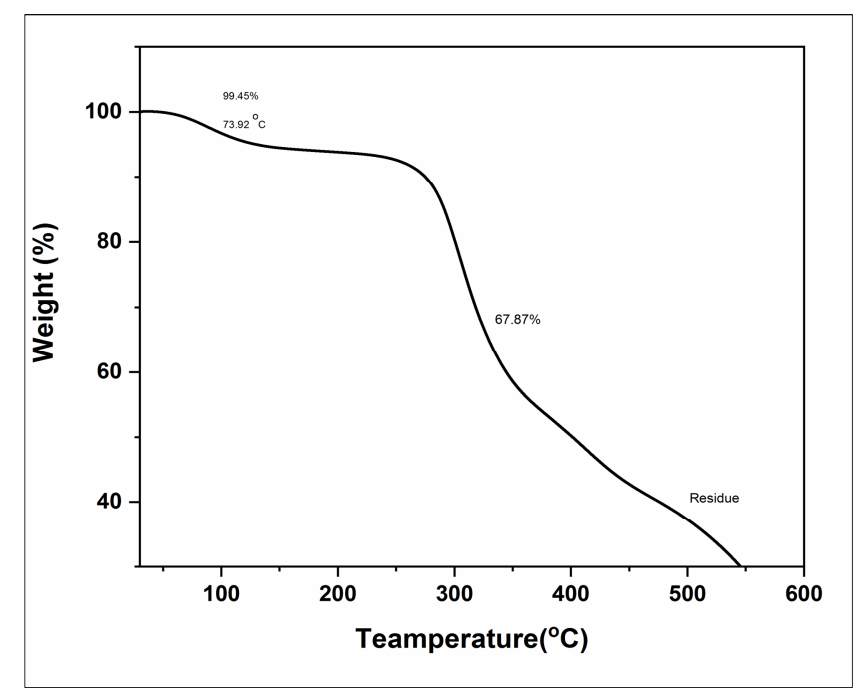

Figure 5. TGA curve of AgNPs loaded silk fibroin hydrogels.

\subsection{In Vitro Release Profiles of AgNPs from Silk Fibroin Hydrogels}

The in vitro release behavior of AgNPs from silk fibroin hydrogels in PBS (pH 7.4) was shown in Figure 6. The AgNPs showed an initial burst release of $44 \pm 3.25 \%, 42 \pm 3.56 \%$, $26 \pm 2.32 \%$ for the formulations F13, F14, and F15, respectively for the first three hours. After that, a sustained release was observed and the cumulative release reached to $69 \pm 1.78 \%, 65 \pm 2.72 \%, 40 \pm 1.42 \%$, respectively for the next 24 hours. Glycerol containing hydrogel showed low initial burst release and prolonged sustained release which might be due to the highly viscous property of glycerol. AgNPs adhered to the surface of fibroin hydrogels might have low mobility to the release medium due to the presence of highly viscous glycerol. Moreover, AgNPs dissolved in glycerol showed reduced gelation time confirming the effect of glycerol in accelerating fibroin gelation kinetics. This could be explained by the fact that glycerol might induce a faster gelation with transforming well-organized $\beta$-sheet structures of fibroin, similar to a crosslinking process, resulting in a more controlled drug 
release $[1,30]$.

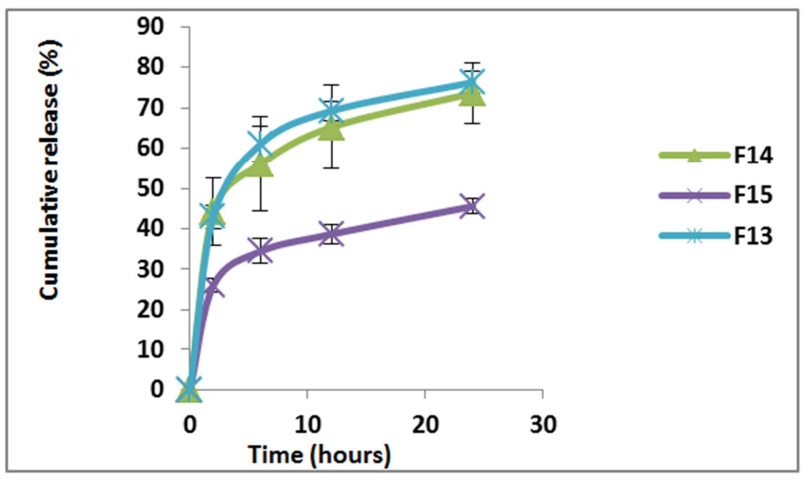

Figure 6. In vitro release profiles of AgNPs from silk fibroin hydrogels.

\subsection{Antifungal Activity of AgNPs Loaded Silk Fibroin Hydrogels}

The antifungal activity of AgNPs loaded silk fibroin hydrogels was tested using a well diffusion method [25]. The antifungal properties of silk fibroin hydrogels loaded with $\mathrm{Ag}$ nanoparticles were analyzed by measuring the zone of inhibition. The diameter of inhibition zones reflects the magnitude of susceptibility of fungus. The strains susceptible to silk fibroin hydrogels incorporated with AgNPs exhibited sharp zone of inhibition. According to the zone of inhibition, Aspergillus Niger exhibited smart sensitivity toward AgNPs incorporated silk fibroin hydrogels. It took 3 days for showing inhibition zone. The result suggests that silk fibroin hydrogels loaded with AgNPs underwent an interaction with fungus cell and displayed the strong action against Aspergillus Niger. The antifungal activity of AgNPs incorporated silk fibroin hydrogels increases with increasing the concentration of AgNPs in hydrogels (Figure 7). A better efficacy was observed when the concentration was $150 \mu \mathrm{g} / \mathrm{mL}$. No significant improvement was observed in efficacy with further increasing the concentration of AgNPs. In contrast, the antifungal activity of silk fibroin hydrogels loaded with AgNPs against pathogen of Trichoderma shows no zone of inhibition.

\section{Conclusion}

In this study, AgNPs loaded silk fibroin hydrogels was prepared by an eco-friendly method. The size of the $\mathrm{Ag}$ nanoparticles was $93 \pm 5 \mathrm{~nm}$ as measured by Zetasizer Nano. Moreover, the absorption peak in the region of 350-400 nm of UV-visible spectra confirmed the formation AgNPs. The prepared AgNPs was incorporated in silk fibroin hydrogels and its morphology, encapsulation efficiency, in vitro release profiles, and antifungal activity was evaluated. Better efficiency and controlled release of AgNPs was observed for glycerol containing formulation F15. The antifungal activity results showed a promising response to Aspergillus Niger, an antifungal microbe. Therefore, the overall results concluded that AgNPs containing silk fibroin hydrogels might be a good candidate for antifungal applications.

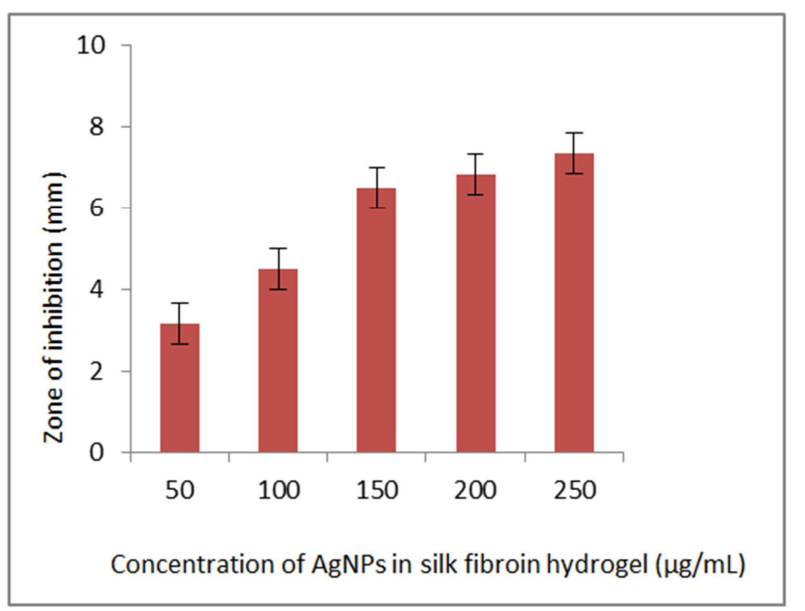

Figure 7. Antifungal activity results of AgNPs loaded silk fibroin hydrogels against Aspergillus Niger.

\section{Acknowledgements}

This research work was supported by the research grant received from Faculty of Science, Rajshahi University, Bangladesh. The authors wish to express their gratitude to the Department of Chemistry, Rajshahi University for providing necessary chemicals and laboratory facilities.

\section{References}

[1] de Moraes M. A., Albrecht C. R., Ferreira S. M., Beppu M. M. (2015) Formation of silk fibroin hydrogel and evaluation of its drug release profile. Journal of Applied Polymer Science, 132: 15 .

[2] Zeng D. M., Pan J. J., Wang Q., Liu X. F., Wang H., Zhang K. Q. (2015) Controlling silk fibroin microspheres via molecular weight distribution. Materials Science Engineering C, 50: 226-233.

[3] Kundu J., Chung Y. I., Kim Y. H., Tae G., Kundu S. C. (2010) Silk fibroin nanoparticles for cellular uptake and control release. International Journal of Pharmaceutics, 388: 242-250.

[4] Ansary R. H., Rahman M. M., Awang M. B., Katas H., Hadi H., Doolaanea A. A. (2016) Preparation, characterization, and in vitro release studies of insulin-loaded double-walled poly (lactide-co-glycolide) microspheres. Drug Delivery and Translational Research, 6 (3): 308-318.

[5] Ansary R. H., Rahman M. M., Awang M. B., Katas H., Hadi H., Mohamed F., Doolaanea A. A., Kamaruzzaman Y. B. (2016) Preparation, characterization and in vitro release study of BSA-loaded double-walled glucose-poly (lactide-co-glycolide) microspheres. Archives of Pharmacal Research, 39 (9): 1242-1256.

[6] Sharma G, Van Der Walle C. F, Kumar M. R. (2013) Antacid co-encapsulated polyester nanoparticles for peroral delivery of insulin: Development, pharmacokinetics, biodistribution and pharmacodynamics. International Journal of Pharmaceutics, 440 (1): 99-110.

[7] Crotts G., Park T. G. (1995) Preparation of porous and nonporous biodegradable polymeric hollow microspheres. Journal of Controlled Release, 35 (2-3): 91-105. 
[8] Ansary R. H., Awang M. B., Rahman M. M. (2014) Biodegradable poly (D, L-lactic-co-glycolic acid)-based micro/nanoparticles for sustained release of protein drugs-A review. Tropical Journal of Pharmaceutical Research, 13 (7): $1179-1190$.

[9] Panyam J., Dali M. M., Sahoo S. K., Ma W., Chakravarthi S. S., Amidon G. La., Levy R. J., Labhasetwar V. (2003) Polymer degradation and in vitro release of a model protein from poly (D, L-lactide-co-glycolide) nano-and microparticles. Journal of Controlled Release, 92 (1-2): 173-187.

[10] Sinha V. R., Trehan A. (2003) Biodegradable microspheres for protein delivery. Journal of Controlled Release, 90 (3): 261-280.

[11] Lu Z., Meng M., Jiang Y., Xie J. (2014) UV-assisted in situ synthesis of silver nanoparticles on silk fibers for antibacterial applications. Colloids and Surfaces: A, 447: 1-7.

[12] Jiang H. L., Jin J. F., Hu Y. Q., Zhu K. J. (2004) Improvement of protein loading and modulation of protein release from poly (lactide-co-glycolide) microspheres by complexation of proteins with polyanions. Journal of Microencapsulation, 21 (6): 615-624.

[13] Zhang Y. Q., Shen W. D., Xiang R. L., Zhuge L. J., Gao W. J., Wang W. B. (2007) Formation of silk fibroin nanoparticles in water-miscible organic solvent and their characterization. Journal of Nanoparticle Research, 9 (5): 885-900.

[14] Gupta V., Aseh A., Ríos C. N., Aggarwal B. B., Mathur A. B. (2009) Fabrication and characterization of silk fibroin-derived curcumin nanoparticles for cancer therapy. International Journal of Nanomedicine, 4: 115.

[15] Calamak S., Aksoy E. A., Ertas N., Erdogdu C., Sagıroglu M., Ulubayram K. (2015) Ag/silk fibroin nanofibers: effect of fibroin morphology on $\mathrm{Ag}+$ release and antibacterial activity. European Polymer Journal, 67: 99-112.

[16] Zhang Q., Yan S., Li M. (2009) Silk fibroin based porous materials. Materials, 2 (4): 2276-2295.

[17] Nagarkar S., Patil A., Lele A., Bhat S., Bellare J., Mashelkar R. A. (2009) Some mechanistic insights into the gelation of regenerated silk fibroin sol. Industrial \& Engineering Chemistry Research, 48 (17): 8014-8023.

[18] Yucel T., Cebe P., Kaplan D. L. (2009) Vortex-induced injectable silk fibroin hydrogels. Biophysical Journal, 97 (7): 2044-2050.

[19] Safdari M., Shakiba E., Kiaie S. H., Fattahi A. (2016) Preparation and characterization of Ceftazidime loaded electrospun silk fibroin/gelatin mat for wound dressing. Fibers and Polymers, 17 (5): 744-750.
[20] Wang Y., Kim H. J., Vunjak-Novakovic G., Kaplan D. L. (2006) Stem cell-based tissue engineering with silk biomaterials. Biomaterials, 27 (36): 6064-6082.

[21] Karageorgiou V., Tomkins M., Fajardo R., Meinel L., Snyder B., Wade K., Chen J., Vunjak - Novakovic G., and Kaplan D. L. (2006) Porous silk fibroin 3 - D scaffolds for delivery of bone morphogenetic protein -2 in vitro and in vivo. Journal of Biomedical Materials Research Part A, 78 (2): 324-334.

[22] Park C. H., Jeong L., Cho D., Kwon O. H., Park W. H. (2013) Effect of methylcellulose on the formation and drug release behavior of silk fibroin hydrogel. Carbohydrate Polymers, 98 (1): 1179-1185

[23] Seib F. P., Pritchard E. M., Kaplan D. L. (2013) Self assembling doxorubicin silk hydrogels for the focal treatment of primary breast cancer. Advanced Functional Materials, 23 (1): 58-65.

[24] Ebrahimi A., Sadrjavadi K., Hajialyani M., Shokoohinia Y., Fattahi A. (2018) Preparation and characterization of silk fibroin hydrogel as injectable implants for sustained release of Risperidone. Drug Develoment and Industrial Pharmacy, 44 (2): 199-205.

[25] Fatema U. K., Rahman M. M., Islam M. R., Mollah M. Y., Susan M. A. (2018) Silver/poly (vinyl alcohol) nanocomposite film prepared using water in oil microemulsion for antibacterial applications. Journal of Colloid and Interface Science, 514: 648-655.

[26] Hanawa T., Watanabe A., Tsuchiya T., Ikoma R., Hidaka M., Sugihara M. (1995) New oral dosage form for elderly patients: preparation and characterization of silk fibroin gel. Chemical and Pharmaceutical Bulletin, 43 (2): 284-288.

[27] Raut R. W., Kolekar N. S., Lakkakula J. R., Mendhulkar V. D., Kashid S. B., (2010) Extracellular synthesis of silver nanoparticles using dried leaves of Pongamia pinnata (L) pierre. Nano-Micro Letters, 2 (2): 106-113.

[28] Veerasamy R., Xin T. Z., Gunasagaran S., Xiang T. F., Yang E. F., Jeyakumar N., Dhanaraj S. A. (2011) Biosynthesis of silver nanoparticles using mangosteen leaf extract and evaluation of their antimicrobial activities. Journal of Saudi Chemical Society, 15 (2): 113-120.

[29] Ribeiro M., de Moraes M. A., Beppu M. M., Monteiro F. J., Ferraz M. P. (2014) The role of dialysis and freezing on structural conformation, thermal properties and morphology of silk fibroin hydrogels. Biomatterials, 4 (1): 28536.

[30] Ansary R. H., Rahman M. M., Mohamad N., Arrif T. M., Latif A. Z., Katas H., Nik W. S., Awang M. B. (2017) Controlled release of lysozyme from double-walled poly (lactide-co-glycolide) (PLGA) microspheres. Polymers, 9 (10): 485 . 